\title{
A MINIMAL UNIFORM RENEWAL THEOREM AND TRANSITION PHENOMENA FOR A NONHOMOGENEOUS PERTURBATION OF THE RENEWAL EQUATION
}

UDC 519.21

\author{
M. V. KARTASHOV
}

\begin{abstract}
We consider the classical continuous time nonhomogeneous renewal equation on the half-line, that is, the integral Volterra equation with a nonnegative bounded kernel. It is assumed that the kernel can be approximated by a convolution kernel generated by a strong substochastic distribution in the large time scale. We study the scheme of series (transition phenomena) where the corresponding defect tends to zero and the time scale tends to infinity. We find the limit of a solution of the renewal equation under certain assumptions. This result is based on a minimal renewal theorem in the scheme of series.

Some applications are also considered.
\end{abstract}

\section{INTRODUCTION}

Risk processes are considered in the papers [2] 11 for the case of a nonhomogeneous environment.

Similarly to the author's preceding paper [3], we study a time nonhomogeneous generalized classical renewal equation on the half-line with continuous time. The equation can be reduced to the integral Volterra equation with a nonnegative bounded (or substochastic) kernel. We assume that, in a large scale, this kernel is approximated in variation by a convolution kernel generated by a strictly substochastic distribution on the positive half-line.

We consider a scheme of series where the total mass of the limit substochastic distribution approaches as the time scale increases (this model corresponds to the well-known scheme of transition phenomena in renewal theory; see [17]).

We prove the existence of a solution of the nonhomogeneous renewal equation and find its limit as the time scale increases.

The proof below uses a minimal renewal theorem in the scheme of series (see Section 2) and is based on some of the methods developed in [12, 13.

\section{A minimal RENEWAL THEOREM IN THE SCHEME OF SERIES}

Consider the half-line $\mathbb{R}_{+}=[0, \infty)$ equipped with the $\sigma$-algebra $\mathfrak{B}_{+}=\mathfrak{B}\left(\mathbb{R}_{+}\right)$.

Introduce the following class of functions:

$\mathrm{B}_{0} \equiv\left\{x: \mathbb{R}_{+} \rightarrow \mathbb{R}\right.$, where $x$ is a Borel function such that $\left.\sup _{s \leq t}|x(s)|<\infty, \forall t \geq 0\right\}$.

2010 Mathematics Subject Classification. Primary 60J45; Secondary 60A05, 60K05.

Key words and phrases. Renewal theory, transition limit theorems, minimal solution, stability, Volterra equations, renewal theorem, regularity, minimal solution, stability.

(C)2011 American Mathematical Society 
To estimate the rate of convergence, we fix a real positive nondecreasing function $\varphi$ defined on $\mathbb{R}_{+}$and such that

$$
\varphi(t+s) \leq \varphi(t) \varphi(s), \quad \lim _{t \rightarrow \infty} \frac{\varphi(t+s)}{\varphi(t)}=1, \quad t, s \geq 0 .
$$

In particular, the functions $1,(1+\ln (1+t))^{\alpha}$ for $\alpha>0,(1+t)^{\beta}$ for $\beta>0$, and $\exp \left(t^{\gamma}\right)$ for $0<\gamma<1$, as well as their multiplicative combinations satisfy all of the above conditions.

Definition 1. We say that a family of measures $\left(G_{\alpha}, \alpha>0\right)$ belongs to the class $\mathrm{L}(\nu, m)$ if there exist an absolutely continuous nonzero measure $\nu$ and a number $m \geq 1$ such that

$$
G_{\alpha}^{* m}(B) \geq \nu(B) \text { for all } \quad \alpha>0 \text { and } B \in \mathfrak{B}_{+} .
$$

Note that the latter condition implies that the distribution $G_{\alpha}$ is of the absolutely continuous type.

Some equivalent conditions of the absolute continuity are presented in [12, 13.

Definition 2. We say that a family of measures $\left(G_{\alpha}, \alpha>0\right)$ belongs to the class $\mathrm{M}(l, \varphi)$ if there exists a function $l(t) \rightarrow 0, t \rightarrow \infty$, such that

$$
\sup _{\alpha} \int_{t}^{\infty} \varphi(s) G_{\alpha}([s, \infty)) d s \leq l(t), \quad t \geq 0 .
$$

Definition 3. Let $t_{\alpha} \rightarrow \infty$ as $\alpha \rightarrow 0$. We say that a family of positive Borel functions $\tau=\left(\tau_{\alpha}(t)\right)$ belongs to the class $\Upsilon_{\infty}\left(\left\{t_{\alpha}\right\}\right)$ if $\tau_{\alpha}(t) \leq t$ and

$$
\inf \left(\tau_{\alpha}(t): \tau_{\alpha}(t)<t \leq t_{\alpha}\right) \rightarrow \infty, \quad \alpha \rightarrow 0 .
$$

Definition 4. For the class of Borel functions $y=\left(y_{\alpha}, \alpha>0\right)$ and for nonnegative functions $\tau=\left(\tau_{\alpha}(t)\right)$ such that $v_{\alpha} \leq t_{\alpha}$, we introduce the following indices:

$$
\begin{gathered}
\sigma_{y, \varphi, \tau}(\alpha, t) \equiv \sup _{0 \leq s<\tau_{\alpha}(t)} \frac{\left|y_{\alpha}(t-s)\right|}{\varphi(s)}, \\
\sigma_{y, \varphi, \tau}\left(\alpha, v_{\alpha}, t_{\alpha}\right) \equiv \sup _{v_{\alpha} \leq t \leq t_{\alpha}} \sigma_{y, \varphi, \tau}(\alpha, t), \\
\lambda_{y, \varphi, \tau}(\alpha, t) \equiv \int_{0 \leq s<\tau_{\alpha}(t)} \frac{\left|y_{\alpha}(t-s)\right|}{\varphi(s)} d s, \\
\lambda_{y, \varphi, \tau}\left(\alpha, v_{\alpha}, t_{\alpha}\right) \equiv \sup _{v_{\alpha} \leq t \leq t_{\alpha}} \lambda_{y, \varphi, \tau}(\alpha, t) .
\end{gathered}
$$

Definition 5. For a function $\varphi$ satisfying (11) and for functions $0 \leq v_{\alpha} \leq t_{\alpha} \rightarrow \infty$, $\alpha \rightarrow 0$, we define the following classes of Borel functions:

$$
\mathrm{B}_{0}^{\varphi}\left(\left\{v_{\alpha}\right\},\left\{t_{\alpha}\right\}\right)=\left\{\begin{array}{c}
y=\left(y_{\alpha}(t)\right): \exists \tau=\left(\tau_{\alpha}(t)\right) \in \Upsilon_{\infty}\left(\left\{t_{\alpha}\right\}\right): \\
\sigma_{y, \varphi, \tau}\left(\alpha, v_{\alpha}, t_{\alpha}\right)=o(1), \sigma_{y, \varphi, t}\left(\alpha, 0, t_{\alpha}\right)=O(1), \alpha \rightarrow 0
\end{array}\right\}
$$

and

$$
\mathrm{L}_{0}^{\varphi}\left(\left\{v_{\alpha}\right\},\left\{t_{\alpha}\right\}\right)=\left\{\begin{array}{c}
y=\left(y_{\alpha}(t)\right): \exists \tau=\left(\tau_{\alpha}(t)\right) \in \Upsilon_{\infty}\left(\left\{t_{\alpha}\right\}\right): \\
\lambda_{y, \varphi, \tau}\left(\alpha, v_{\alpha}, t_{\alpha}\right)=o(1), \lambda_{y, \varphi, t}\left(\alpha, 0, t_{\alpha}\right)=O(1), \alpha \rightarrow 0
\end{array}\right\} .
$$

Theorem 1. Let, for each $\alpha>0$, a function $x_{\alpha} \in \mathrm{B}_{0}$ satisfy the renewal equation with $y_{\alpha} \in \mathrm{B}_{0}$, namely

$$
x_{\alpha}(t)=y_{\alpha}(t)+x_{\alpha} * F_{\alpha}(t) \equiv y_{\alpha}(t)+\int_{[0, t)} x_{\alpha}(t-s) F_{\alpha}(d s), \quad t \geq 0,
$$


where the members of the family of probability distributions $\left(F_{\alpha}\right)$ have the mean values $m_{\alpha} \equiv \int_{0}^{\infty} s F_{\alpha}(d s)$ and are such that

$$
\left(F_{\alpha}\right) \subset \mathrm{L}(\nu, m) \cap \mathrm{M}(l, \varphi)
$$

(the classes $\mathrm{L}(\nu, m)$ and $\mathrm{M}(l, \varphi)$ are introduced in Definitions 1 and 2, respectively).

If the functions are such that $0 \leq v_{\alpha} \leq t_{\alpha} \rightarrow \infty$ as $\alpha \rightarrow 0$ and

$$
\left(y_{\alpha}\right) \subset \mathrm{B}_{0}^{\varphi}\left(\left\{v_{\alpha}\right\},\left\{t_{\alpha}\right\}\right) \cap \mathrm{L}_{0}^{\varphi}\left(\left\{v_{\alpha}\right\},\left\{t_{\alpha}\right\}\right),
$$

then

$$
\begin{aligned}
\sup _{v_{\alpha} \leq t \leq t_{\alpha}}\left|x_{\alpha}(t)-m_{\alpha}^{-1} \int_{0}^{t} y_{\alpha}(s) d s\right| \\
=O\left(\sigma_{y, \varphi, \tau}\left(\alpha, v_{\alpha}, t_{\alpha}\right)\right)+o\left(\sigma_{y, \varphi, t}\left(\alpha, 0, t_{\alpha}\right)\right) \\
\quad+O\left(\lambda_{y, \varphi, \tau}\left(\alpha, v_{\alpha}, t_{\alpha}\right)\right)+o\left(\lambda_{y, \varphi, t}\left(\alpha, 0, t_{\alpha}\right)\right) \rightarrow 0, \quad \alpha \rightarrow 0,
\end{aligned}
$$

according to definitions (3) and (4).

Remark 1. The first condition in definition (4) follows from the first condition in definition (3) if $v_{\alpha} \equiv 0$.

We need the following definition to study the rate of convergence in (6).

Definition 6. Assume that a function $\varphi$ satisfies (11) and that the functions $v_{\alpha}$ are such that $0 \leq v_{\alpha} \leq t_{\alpha} \rightarrow \infty$ as $\alpha \rightarrow 0$. We introduce the classes of Borel functions $\overline{\mathrm{B}}_{0}^{\varphi}\left(\left\{v_{\alpha}\right\},\left\{t_{\alpha}\right\}\right)$ and $\overline{\mathrm{L}}_{0}^{\varphi}\left(\left\{v_{\alpha}\right\},\left\{t_{\alpha}\right\}\right)$ according to relations (3) and (4), respectively, where the indices (2) are changed by

$$
\bar{\sigma}_{y, \varphi, \tau}(\alpha, t) \equiv \sup _{0 \leq s<\tau_{\alpha}(t)}\left|y_{\alpha}(t-s)\right| \varphi(t-s)
$$

and

$$
\bar{\lambda}_{y, \varphi, \tau}(\alpha, t) \equiv \int_{0 \leq s<\tau_{\alpha}(t)}\left|y_{\alpha}(t-s)\right| \varphi(t-s) d s,
$$

respectively.

Theorem 2. Let the assumptions of Theorem 1 hold with $\overline{\mathrm{B}}_{0}^{\varphi}$ and $\overline{\mathrm{L}}_{0}^{\varphi}$ instead of $\mathrm{B}_{0}^{\varphi}$ and $\mathrm{L}_{0}^{\varphi}$, respectively. Then

$$
\sup _{v_{\alpha} \leq t \leq t_{\alpha}} \varphi(t)\left|x_{\alpha}(t)-m_{\alpha}^{-1} \int_{0}^{t} y_{\alpha}(s) d s\right| \rightarrow 0, \quad \alpha \rightarrow 0 .
$$

\section{Perturbed transition Renewal equation}

Let $\left(G_{\alpha}, \alpha>0\right)$ be a family of strictly substochastic measures on $\mathfrak{B}_{+}$.

The integral equation

$$
x_{\alpha}(t)=y_{\alpha}(t)+\int_{[0, t)} x_{\alpha}(t-s) G_{\alpha}(d s), \quad t \geq 0,
$$

with respect to an unknown function $x_{\alpha} \in \mathrm{B}_{0}$ is called an improper renewal equation on $\mathbb{R}_{+}$generated by a family of strictly substochastic measures $\left(G_{\alpha}\right)$.

Let $\left(F_{\alpha}(t, B), t \geq 0, B \in \mathfrak{B}_{+}, \alpha>0\right)$ be a family of bounded nonnegative kernels.

Definition 7. The integral Volterra equation

$$
x_{\alpha}(t)=y_{\alpha}(t)+\int_{[0, t)} x_{\alpha}(t-s) F_{\alpha}(t, d s), \quad t \geq 0,
$$

with respect to an unknown function $x_{\alpha} \in \mathrm{B}_{0}$ and a given function $y_{\alpha}$ is called a nonhomogeneous perturbation of the renewal equation (9). 
The kernel $F_{\alpha}$ in equation (10) approximates the measure $G_{\alpha}$ in variation:

$$
\Delta_{\alpha}(t, B) \equiv F_{\alpha}(t, B)-G_{\alpha}(B) \rightarrow 0, \quad t \rightarrow \infty
$$

uniformly with respect to $\alpha$ and $B \in \mathfrak{B}_{+} \cap[0, t)$. This means that the perturbation function $\delta_{\alpha}(t)$ is such that

$$
\delta_{\alpha}(t) \equiv\left|\Delta_{\alpha}\right|(t,[0, t))=\sup _{|x| \leq 1}\left|\int_{0}^{t} x(s) \Delta_{\alpha}(t, d s)\right| \rightarrow 0, \quad t \rightarrow \infty .
$$

Assume that the following extended Cramér condition holds:

$$
\forall \alpha>0 \exists \delta>0: \quad \widehat{g}_{\alpha}(\delta) \equiv \int_{0}^{\infty} \exp (\delta x) G_{\alpha}(d x) \in(1, \infty) .
$$

The Lundberg index

$$
\beta=\beta(\alpha) \equiv \sup \left(s \geq 0: \widehat{g}_{\alpha}(s) \leq 1\right)
$$

is finite under the Cramér condition (13). At the same time, the Lundberg index is a unique positive root of the equation

$$
\widehat{g}_{\alpha}(\beta)=1
$$

and moreover

$$
\mu_{\alpha} \equiv \widehat{g}_{\alpha}^{\prime}(\beta)=\int_{0}^{\infty} x \exp (\beta x) G_{\alpha}(d x)<\infty .
$$

This implies that the corresponding exponential transform of $G_{\alpha}$ is a probability measure; namely,

$$
\bar{G}_{\alpha}(B) \equiv \int_{B} \exp (\beta s) G_{\alpha}(d s), \quad B \in \mathfrak{B}_{+},
$$

where $\bar{G}_{\alpha}\left(\mathbb{R}_{+}\right)=1$.

The transition phenomenon in the renewal theory [17] means that $G_{\alpha}\left(\mathbb{R}_{+}\right) \rightarrow 1$ as $\alpha \rightarrow 0$. This assumption implies the following convergences:

$$
\beta \rightarrow 0, \quad \frac{\beta \mu_{\alpha}}{\left(1-G_{\alpha}\left(\mathbb{R}_{+}\right)\right)} \rightarrow 1, \quad \alpha \rightarrow 0 .
$$

In what follows we consider a scheme with the function $\varphi$ in (11) and (2) being equal to 1 for all arguments.

Theorem 3. Let a family $\left(x_{\alpha}\right) \subset \mathrm{B}_{0}$ be a solution of the nonhomogeneous perturbation (10) of the improper renewal equation (9). Further let a family of kernels $\left(F_{\alpha}(t, B)\right)$ be a perturbation (11) of strictly substochastic measures $\left(G_{\alpha}(B)\right)$ and let the Cramér condition (13) hold. Assume that the generated probabilities (15) are such that

$$
\left(\bar{G}_{\alpha}\right) \subset \mathrm{L}(\nu, m) \cap \mathrm{M}(l, 1) .
$$

Let $\alpha \rightarrow 0$ and let the functions $0 \leq v_{\alpha} \leq t_{\alpha} \rightarrow \infty$ be such that $\beta t_{\alpha} \rightarrow u>0$, where the index $\beta$ is defined in (14). Assume also that

$$
\begin{aligned}
& \left(y_{\alpha}\right) \subset \mathrm{B}_{0}^{1}\left(\left\{v_{\alpha}\right\},\left\{t_{\alpha}\right\}\right) \cap \mathrm{L}_{0}^{1}\left(\left\{v_{\alpha}\right\},\left\{t_{\alpha}\right\}\right), \\
& \left(\delta_{\alpha}\right) \subset \mathrm{B}_{0}^{1}\left(\left\{v_{\alpha}\right\},\left\{t_{\alpha}\right\}\right) \cap \mathrm{L}_{0}^{1}\left(\left\{v_{\alpha}\right\},\left\{t_{\alpha}\right\}\right),
\end{aligned}
$$

and that the solutions $\left(x_{\alpha}\right)$ are locally bounded in the sense that

$$
\sup _{\alpha>0} \sup _{t \leq t_{\alpha}}\left|x_{\alpha}(t)\right|<\infty
$$


Finally we assume that

$$
\begin{gathered}
\rho_{\alpha} \equiv \int_{0}^{t_{\alpha}}\left|\Delta_{\alpha}\right|\left(t,\left[t-v_{\alpha}, t\right)\right) d t \rightarrow 0, \quad \alpha \rightarrow 0, \\
\varepsilon_{\alpha} \equiv \int_{0}^{t_{\alpha}} \delta_{\alpha}(s) d s \int_{s}^{t_{\alpha}}\left|\Delta_{\alpha}\right|(t,[0, t-s)) d t \rightarrow 0, \quad \alpha \rightarrow 0 .
\end{gathered}
$$

Then the representation

$$
\begin{gathered}
e^{\beta t} x_{\alpha}(t)-\mu_{\alpha}^{-1} \int_{0}^{t}\left[e^{\beta s} y_{\alpha}(s)+\mu_{\alpha}^{-1} \int_{0}^{s} e^{\beta u} y_{\alpha}(u) \int_{0}^{s-u} e^{\beta v} \Delta_{\alpha}(s, d v) d u\right] d s \\
=O\left(\gamma_{\alpha}+\rho_{\alpha}+\varepsilon_{\alpha}\right)+o\left(\Gamma_{\alpha}\right) \rightarrow 0, \quad \alpha \rightarrow 0,
\end{gathered}
$$

holds uniformly with respect to $t \in\left[v_{\alpha}, t_{\alpha}\right]$, where

$$
\begin{aligned}
\gamma_{\alpha} \equiv & \sigma_{y, \varphi, \tau}\left(\alpha, v_{\alpha}, t_{\alpha}\right)+\lambda_{y, \varphi, \tau}\left(\alpha, v_{\alpha}, t_{\alpha}\right) \\
& +\sigma_{\delta, \varphi, \tau}\left(\alpha, v_{\alpha}, t_{\alpha}\right)+\lambda_{\delta, \varphi, \tau}\left(\alpha, v_{\alpha}, t_{\alpha}\right)=o(1), \quad \alpha \rightarrow 0, \\
\Gamma_{\alpha} \equiv & \sigma_{y, \varphi, t}\left(\alpha, 0, t_{\alpha}\right)+\lambda_{y, \varphi, t}\left(\alpha, 0, t_{\alpha}\right) \\
& +\sigma_{\delta, \varphi, t}\left(\alpha, 0, t_{\alpha}\right)+\lambda_{\delta, \varphi, t}\left(\alpha, 0, t_{\alpha}\right)=O(1), \quad \alpha \rightarrow 0,
\end{aligned}
$$

by (3) and (44). All other symbols are defined in (2).

Remark 2. Condition (19) holds if $x_{\alpha}(t)$ is the probability of a certain random event. Moreover, condition (19) holds if

$$
\sup _{\alpha>0, t \geq 0} \frac{\left|y_{\alpha}(t)\right|}{1-F_{\alpha}(t,[0, t))}<\infty .
$$

Corollary 1. Let a family $\left(x_{\alpha}\right) \subset \mathrm{B}_{0}$ be a solution of an improper renewal equation (9) with strictly substochastic measures $\left(G_{\alpha}\right)$. Assume that Cramér's condition (13) holds and that the generated probabilities (15) are such that

$$
\left(\bar{G}_{\alpha}\right) \subset \mathrm{L}(\nu, m) \cap \mathrm{M}(l, 1) .
$$

Let $\alpha \rightarrow 0$ and the functions $0 \leq v_{\alpha} \leq t_{\alpha} \rightarrow \infty$ be such that $\beta t_{\alpha} \rightarrow u>0$, where $\beta$ is defined by (14). We also assume that

$$
\left(y_{\alpha}\right) \subset \mathrm{B}_{0}^{1}\left(\left\{v_{\alpha}\right\},\left\{t_{\alpha}\right\}\right) \cap \mathrm{L}_{0}^{1}\left(\left\{v_{\alpha}\right\},\left\{t_{\alpha}\right\}\right) .
$$

If the solutions $\left(x_{\alpha}\right)$ are locally bounded, that is,

$$
\sup _{\alpha>0} \sup _{t \leq t_{\alpha}}\left|x_{\alpha}(t)\right|<\infty
$$

then

$$
x_{\alpha}(t)-\mu_{\alpha}^{-1} \int_{0}^{t} e^{-\beta(t-s)} y_{\alpha}(s) d s \rightarrow 0, \quad \alpha \rightarrow 0,
$$

uniformly with respect to $t \in\left[v_{\alpha}, t_{\alpha}\right]$.

\section{An application to the Claim processes}

In the paper [3, we considered a risk process modeled by the underlying ruin probabilities at sequential claim moments. Below we describe an application of the limit theorem for the perturbed transition renewal equation to the asymptotic analysis of this model of risk processes.

Consider an insurance company with an initial capital $t>0$ and assume that the claims $\left(\xi_{k}, k \geq 1\right)$ are independent and have a common distribution function $G(t)=\mathrm{P}\left(\xi_{1}<t\right)$ of an absolutely continuous type. Put $S_{n}=\xi_{1}+\cdots+\xi_{n}, S_{0}=0$, and let $\mu=\mathrm{E} \xi_{1}$. 
Assume that the insurance company ruins with probability $\pi_{\alpha}\left(t-S_{k-1}\right)$ between the $(k-1)$-th and $k$-th payments (the company does continue functioning with probability $\left.1-\pi_{\alpha}\left(t-S_{k-1}\right)\right)$. Note that the probability $\pi_{\alpha}\left(t-S_{k-1}\right)$ depends on the current capital of the company available between the $(k-1)$-th and $k$-th payments. Here $\alpha>0$ stands for the parameter of the scheme of series.

We assume that the ruin is independent of the sequence of claims given the rest of the capital of the company at a corresponding claim moment.

Denote by $\nu(t)$ the total number of claims that occurred prior to the ruin of the insurance company. Then

$$
\mathrm{P}_{\alpha}\left(\nu(t)=n \mid S_{0}, \ldots, S_{n}\right)=\left[\prod_{k=0}^{n-1}\left(1-\pi_{\alpha}\left(t-S_{k}\right)\right)\right] \pi_{\alpha}\left(t-S_{n}\right) 1_{\left\{S_{n}<t\right\}}, \quad n \geq 0 .
$$

The distribution function of the rest of the capital of the insurance company at the moment of ruin is given by

$$
x_{\alpha}(t, b)=\mathrm{P}_{\alpha}\left(t-b \leq S_{\nu(t)}<t\right)=\mathrm{P}_{\alpha}\left(0<t-S_{\nu(t)} \leq b\right)
$$

for $b<t$.

This distribution function is a solution of the nonhomogeneous transition renewal equation

$$
x_{\alpha}(t, b)=\pi_{\alpha}(t) 1_{t \leq b}+\left(1-\pi_{\alpha}(t)\right) \int_{0}^{t} x_{\alpha}(t-s, b) G(d s) .
$$

Assume that the limit

$$
\theta_{\alpha} \equiv \lim _{t \rightarrow \infty} \pi_{\alpha}(t) \in(0,1)
$$

exists for all $\alpha>0$.

Equation (25) coincides with (10) if

$$
F_{\alpha}(t, d s) \equiv\left(1-\pi_{\alpha}(t)\right) G(d s) 1_{s<t}, \quad y_{\alpha}(t)=\pi_{\alpha}(t) 1_{t \leq b} .
$$

The kernel $\left(F_{\alpha}\right)$ satisfies condition (11) with the measure

$$
G_{\alpha}=\left(1-\theta_{\alpha}\right) G,
$$

where the perturbation (12) is of the form

$$
\Delta_{\alpha}(t, d s)=-\nu_{\alpha}(t) G(d s) 1_{s<t}, \quad \delta_{\alpha}(t)=\left|\nu_{\alpha}(t)\right| G(t),
$$

with the perturbation function

$$
\nu_{\alpha}(t) \equiv \pi_{\alpha}(t)-\theta_{\alpha}
$$

We assume that

$$
\theta_{\alpha} \rightarrow 0, \quad \alpha \rightarrow 0
$$

in the scheme of transition phenomena.

Let the following Cramér condition hold for the amount of a single claim; namely, let a random variable $\xi_{1}$ be such that

$$
\exists \delta>0: \quad \widehat{G}(\delta) \equiv \mathrm{E} \exp \left(\delta \xi_{1}\right) \in(1, \infty) .
$$

The Lundberg index is defined by

$$
\beta=\beta(\alpha) \equiv \sup \left(s \geq 0:\left(1-\theta_{\alpha}\right) \widehat{G}(s) \leq 1\right) .
$$

Note that the Lundberg index is a unique positive solution of the equation

$$
\left(1-\theta_{\alpha}\right) \widehat{G}(\beta)=1 \text {. }
$$


Note also that $\beta \rightarrow 0$ as $\alpha \rightarrow 0$ and moreover

$$
\frac{\theta_{\alpha}}{\beta} \rightarrow \mu=\mathrm{E} \xi_{1}, \quad \alpha \rightarrow 0
$$

under the condition (31).

Theorem 4. Let the distribution function $G$ be of the absolutely continuous type and satisfy Cramér's condition (32).

Let the limit relations (26) and (31) hold for the ruin probability and moreover let $\alpha \rightarrow 0$ and $b_{\alpha} \rightarrow \infty$ as $t_{\alpha} \rightarrow \infty$ in such a way that $\beta t_{\alpha} \rightarrow u>0$ and $\beta b_{\alpha} \rightarrow v \in(0, u)$. We also assume that the perturbation (30) is such that

$$
\begin{aligned}
\psi_{\alpha} & \equiv \sup _{0 \leq t<t_{\alpha}}\left|\nu_{\alpha}(t)\right|=o(1), \quad \alpha \rightarrow 0, \\
\phi_{\alpha} & \equiv \int_{0}^{t_{\alpha}}\left|\nu_{\alpha}(s)\right| d s=o(1), \quad \alpha \rightarrow 0 .
\end{aligned}
$$

Then

$$
\begin{gathered}
x_{\alpha}\left(t_{\alpha}, b_{\alpha}\right)-e^{-u+v}+e^{-u}+e^{-u} \mu^{-1} \int_{0}^{u} \nu_{\alpha}(t / \beta) \beta^{-1}\left[e^{t} 1_{t \leq v}-e^{v}+1\right] d t \\
=o\left(\theta_{\alpha}\right)+O\left(\phi_{\alpha}\right)+O\left(\psi_{\alpha}\right) \rightarrow 0, \quad \alpha \rightarrow 0 .
\end{gathered}
$$

Example 1. Let $\pi_{\alpha}(t)=\theta_{\alpha}(1+l(t))$, where the function $l(t)>-1$ is integrable and bounded. Then conditions (36) and (37) hold and

$$
x_{\alpha}\left(t_{\alpha}, b_{\alpha}\right) \rightarrow \exp (-u+v)-\exp (-u), \quad \alpha \rightarrow 0 .
$$

Example 2. Let $\pi_{\alpha}(t)=\theta_{\alpha}+\varkappa_{\alpha} \beta l(\beta t)$, where $0<\varkappa_{\alpha} \rightarrow 0$ is such that $\theta_{\alpha}=o\left(\varkappa_{\alpha}\right)$ as $\alpha \rightarrow 0$, while the function $l(t)>-\inf _{\alpha>0} \theta_{\alpha} / \varkappa_{\alpha} \beta$ is integrable and bounded. Then conditions (36) and (37) hold and

$$
x_{\alpha}\left(t_{\alpha}, b_{\alpha}\right)-e^{-u+v}+e^{-u}+\varkappa_{\alpha} e^{-u} \mu^{-1} \int_{0}^{u} l(t)\left[e^{t} 1_{t \leq v}-e^{v}+1\right] d t=o\left(\varkappa_{\alpha}\right), \quad \alpha \rightarrow 0 .
$$

\section{Proofs}

Proof of Theorem 1. Let $x_{\alpha} \in \mathrm{B}_{0}$ be a solution of equation (9). This solution is unique and is given by

$$
x_{\alpha}(t)=y_{\alpha} * U_{\alpha}(t) \equiv \int_{0}^{t} y_{\alpha}(t-s) U_{\alpha}(d s)
$$

(see [14]-[16]), where $U_{\alpha}$ is the renewal measure,

$$
U_{\alpha}(B)=\sum_{n \geq 0} F_{\alpha}^{* n}(B), \quad B \in \mathfrak{B}_{+} .
$$

The inclusion $\left(F_{\alpha}\right) \subset \mathrm{L}(\nu, m) \cap \mathrm{M}(l, \varphi)$ implies that the assumptions of Theorem 2 in [13] hold for the class $\left(F_{\alpha}\right)$. Thus we obtain the Stone representation $U_{\alpha}=V_{\alpha}+H_{\alpha}$, and moreover,

$$
\sup _{\alpha} \int_{0}^{\infty} \varphi(s) V_{\alpha}(d s)<\infty, \quad \lim _{t \rightarrow \infty} \sup _{\alpha} \int_{t}^{\infty} \varphi(s) V_{\alpha}([s, \infty)) d s=0 .
$$

The measure $H_{\alpha}$ possesses the density $h_{\alpha}$ such that

$$
\sup _{\alpha, t} \varphi(t)\left|h_{\alpha}(t)-m_{\alpha}^{-1}\right|<\infty, \quad \lim _{t \rightarrow \infty} \varphi(t) \sup _{\alpha}\left|h_{\alpha}(t)-m_{\alpha}^{-1}\right|=0,
$$


where the family of the mean values

$$
m_{\alpha} \equiv \int_{0}^{\infty} t F_{\alpha}(d t) \geq m^{-1} \int_{0}^{\infty} t \nu(d t)>0
$$

is bounded away from zero by Definition 1 .

In particular,

$$
x_{\alpha}(t)-m_{\alpha}^{-1} \int_{0}^{t} y_{\alpha}(s) d s=y_{\alpha} * V_{\alpha}(t)+y_{\alpha} *\left(h_{\alpha}-m_{\alpha}^{-1}\right)(t) .
$$

Put $\tau(\alpha) \equiv \inf \left(\tau_{\alpha}(t): \tau_{\alpha}(t)<t \leq t_{\alpha}\right)$. Then

$$
\tau(\alpha) \rightarrow \infty, \quad \alpha \rightarrow 0,
$$

in view of the inclusion $\tau=\left(\tau_{\alpha}(t)\right) \in \Upsilon_{\infty}\left(\left\{t_{\alpha}\right\}\right)$.

Lemma 1. Let a family of measures $\left(V_{\alpha}\right)$ satisfy conditions (39) and

$$
0 \leq v_{\alpha} \leq t_{\alpha} \rightarrow \infty \quad \text { as } \alpha \rightarrow 0 .
$$

(a) If $\left(y_{\alpha}\right) \subset \mathrm{B}_{0}^{\varphi}\left(\left\{v_{\alpha}\right\},\left\{t_{\alpha}\right\}\right)$, then

$$
\sup _{v_{\alpha} \leq t \leq t_{\alpha}}\left|y_{\alpha} * V_{\alpha}(t)\right|=O\left(\sigma_{y, \varphi, \tau}\left(\alpha, v_{\alpha}, t_{\alpha}\right)\right)+o\left(\sigma_{y, \varphi, t}\left(\alpha, 0, t_{\alpha}\right)\right), \quad \alpha \rightarrow 0 .
$$

(b) If $\left(y_{\alpha}\right) \subset \overline{\mathrm{B}}_{0}^{\varphi}\left(\left\{v_{\alpha}\right\},\left\{t_{\alpha}\right\}\right)$, then

$$
\sup _{v_{\alpha} \leq t \leq t_{\alpha}} \varphi(t)\left|y_{\alpha} * V_{\alpha}(t)\right| \rightarrow 0, \quad \alpha \rightarrow 0
$$

Proof. For statement (a), we have

$$
\begin{aligned}
\sup _{v_{\alpha} \leq t \leq t_{\alpha}}\left|y_{\alpha} * V_{\alpha}(t)\right| \leq & \sup _{v_{\alpha} \leq t \leq t_{\alpha}} \int_{0}^{\tau_{\alpha}(t)}\left|y_{\alpha}(t-s) / \varphi(s)\right| \varphi(s) V_{\alpha}(d s) \\
& +\sup _{t \leq t_{\alpha}} \int_{\tau_{\alpha}(t)}^{t}\left|y_{\alpha}(t-s) / \varphi(s)\right| \varphi(s) V_{\alpha}(d s) \\
\leq & \sup _{v_{\alpha} \leq t \leq t_{\alpha}} \sigma_{y, \varphi, \tau}(\alpha, t) \sup _{v_{\alpha} \leq t \leq t_{\alpha}} \int_{0}^{t} \varphi(s) V_{\alpha}(d s) \\
& +\sup _{t \leq t_{\alpha}} \sigma_{y, \varphi, t}(\alpha, t) \int_{\tau(\alpha)}^{t_{\alpha}} \varphi(s) V_{\alpha}(d s) \rightarrow 0, \quad \alpha \rightarrow 0,
\end{aligned}
$$

in view of (2), (3), and (43).

For statement (b), we use properties (10) and obtain

$$
\begin{aligned}
\sup _{v_{\alpha} \leq t \leq t_{\alpha}} \varphi(t)\left|y_{\alpha} * V_{\alpha}(t)\right| \leq & \sup _{v_{\alpha} \leq t \leq t_{\alpha}} \int_{0}^{\tau_{\alpha}(t)}\left|y_{\alpha}(t-s) \varphi(t-s)\right| \varphi(s) V_{\alpha}(d s) \\
& +\sup _{t \leq t_{\alpha}} \int_{\tau_{\alpha}(t)}^{t}\left|y_{\alpha}(t-s) \varphi(t-s)\right| \varphi(s) V_{\alpha}(d s) \\
\leq & \sup _{v_{\alpha} \leq t \leq t_{\alpha}} \bar{\sigma}_{y, \varphi, \tau}(\alpha, t) \sup _{v_{\alpha} \leq t \leq t_{\alpha}} \int_{0}^{\tau_{\alpha}(t)} \varphi(s) V_{\alpha}(d s) \\
& +\sup _{t \leq t_{\alpha}} \bar{\sigma}_{y, \varphi, t}(\alpha, t) \int_{\tau(\alpha)}^{t_{\alpha}} \varphi(s) V_{\alpha}(d s) \rightarrow 0, \quad \alpha \rightarrow 0,
\end{aligned}
$$

in view of (7) and (43). 
Lemma 2. Let the densities $\left(h_{\alpha}\right)$ satisfy conditions (40) and let $0 \leq v_{\alpha} \leq t_{\alpha} \rightarrow \infty$ as $\alpha \rightarrow 0$.

(a) If $\left(y_{\alpha}\right) \subset \mathrm{L}_{0}^{\varphi}\left(\left\{v_{\alpha}\right\},\left\{t_{\alpha}\right\}\right)$, then

$$
\sup _{v_{\alpha} \leq t \leq t_{\alpha}}\left|y_{\alpha} *\left(h_{\alpha}-m_{\alpha}^{-1}\right)(t)\right|=O\left(\lambda_{y, \varphi, \tau}\left(\alpha, v_{\alpha}, t_{\alpha}\right)\right)+o\left(\lambda_{y, \varphi, t}\left(\alpha, 0, t_{\alpha}\right)\right), \quad \alpha \rightarrow 0 .
$$

(b) If $\left(y_{\alpha}\right) \subset \overline{\mathrm{L}}_{0}^{\varphi}\left(\left\{v_{\alpha}\right\},\left\{t_{\alpha}\right\}\right)$, then

$$
\sup _{v_{\alpha} \leq t \leq t_{\alpha}} \varphi(t)\left|y_{\alpha} *\left(h_{\alpha}-m_{\alpha}^{-1}\right)(t)\right| \rightarrow 0, \quad \alpha \rightarrow 0 .
$$

Proof. In the case of (a), definition (4) implies that

$$
\begin{aligned}
\sup _{v_{\alpha} \leq t \leq t_{\alpha}}\left|y_{\alpha} *\left(h_{\alpha}-m_{\alpha}^{-1}\right)(t)\right| \\
=\sup _{v_{\alpha} \leq t \leq t_{\alpha}}\left|\int_{0}^{t} y_{\alpha}(t-s)\left[h_{\alpha}(s)-m_{\alpha}^{-1}\right] d s\right| \\
\leq \sup _{v_{\alpha} \leq t \leq t_{\alpha}} \int_{0}^{\tau_{\alpha}(t)}\left|y_{\alpha}(t-s) / \varphi(s)\right| \varphi(s)\left|h_{\alpha}(s)-m_{\alpha}^{-1}\right| d s \\
\leq \sup _{t \leq t_{\alpha}} \int_{\tau_{\alpha}(t)}^{t}\left|y_{\alpha}(t-s) / \varphi(s)\right| \varphi(s)\left|h_{\alpha}(s)-m_{\alpha}^{-1}\right| d s \\
\leq \sup _{v_{\alpha} \leq t \leq t_{\alpha}} \lambda_{y, \varphi, \tau}(\alpha, t) \sup _{s \geq 0} \varphi(s)\left|h_{\alpha}(s)-m_{\alpha}^{-1}\right| \\
\quad+\sup _{t \leq t_{\alpha}} \lambda_{y, \varphi, t}(\alpha, t) \sup _{s \geq \tau(\alpha)} \varphi(s)\left|h_{\alpha}(s)-m_{\alpha}^{-1}\right| \rightarrow 0, \quad \alpha \rightarrow 0,
\end{aligned}
$$

in view of (43).

In the case of (b), we derive from (10),

$$
\begin{aligned}
\sup _{v_{\alpha} \leq t \leq t_{\alpha}} & \varphi(t)\left|y_{\alpha} *\left(h_{\alpha}-m_{\alpha}^{-1}\right)(t)\right| \\
= & \sup _{v_{\alpha} \leq t \leq t_{\alpha}} \varphi(t)\left|\int_{0}^{t} y_{\alpha}(t-s)\left[h_{\alpha}(s)-m_{\alpha}^{-1}\right] d s\right| \\
\leq & \sup _{v_{\alpha} \leq t \leq t_{\alpha}} \int_{0}^{\tau_{\alpha}(t)}\left|y_{\alpha}(t-s) \varphi(t-s)\right| \varphi(s)\left|h_{\alpha}(s)-m_{\alpha}^{-1}\right| d s \\
& \quad+\sup _{t \leq t_{\alpha}} \int_{\tau_{\alpha}(t)}^{t}\left|y_{\alpha}(t-s) \varphi(t-s)\right| \varphi(s)\left|h_{\alpha}(s)-m_{\alpha}^{-1}\right| d s \\
\leq & \sup _{v_{\alpha} \leq t \leq t_{\alpha}} \bar{\lambda}_{y, \varphi, \tau}(\alpha, t) \sup _{s \geq 0} \varphi(s)\left|h_{\alpha}(s)-m_{\alpha}^{-1}\right| \\
& +\sup _{t \leq t_{\alpha}} \bar{\lambda}_{y, \varphi, t}(\alpha, t) \sup _{s \geq \tau(\alpha)} \varphi(s)\left|h_{\alpha}(s)-m_{\alpha}^{-1}\right| \rightarrow 0, \quad \alpha \rightarrow 0,
\end{aligned}
$$

in view of (43).

Applying Lemmas 1(a) and 2(a) to (42) and taking into account equality (41), we complete the proof of Theorem 1.

To prove Remark 1 we choose

$$
\tau_{\alpha}(t)=\min \left\{\tau_{\alpha}(t),\left(\sup _{0 \leq t \leq t_{\alpha}} \sigma_{y, \varphi, \tau}(\alpha, t)\right)^{-1 / 2}\right\}
$$

in definition (4), where the function $\tau=\left(\tau_{\alpha}(t)\right)$ on the right hand side is defined by (3). 
Now we estimate the integrand on the right hand side of (2), defining $\lambda_{y, \varphi, \tau}(\alpha, t)$ by its supremum over the interval $\left[0, \tau_{\alpha}(t)\right)$. Then we use the result in definition (4) of $\lambda_{y, \varphi, \tau}\left(\alpha, v_{\alpha}, t_{\alpha}\right)$.

Proof of Theorem 2. The proof is completely similar to the preceding one and easily follows from representation (42) and Lemmas 1(b) and 2(b).

Proof of Theorem 3. Equality (14) follows from (13), since the function $\widehat{g}_{\alpha}(s)$ is continuous and bounded for $s \in[0, \delta]$.

The equivalence of functions in (16) follows from the mean value theorem and equality (14):

$$
\frac{\beta \mu_{\alpha}}{1-G_{\alpha}\left(\mathbb{R}_{+}\right)}=\frac{\beta \mu_{\alpha}}{\widehat{g}_{\alpha}(\beta)-\widehat{g}_{\alpha}(0)}=\frac{\beta \mu_{\alpha}}{\beta \widehat{g}_{\alpha}^{\prime}(\widetilde{\beta})} \rightarrow 1, \quad \alpha \rightarrow 0 .
$$

The convergence $\gamma_{\alpha} \rightarrow 0$ as $\alpha \rightarrow 0$ in relation (22) follows from relations (18), (3), and (4).

For a kernel $F$, we introduce the following linear bounded operator on $\mathrm{B}_{0}$ :

$$
F[x](t)=\int_{0}^{t} F(t, d s) x(t-s), \quad t \geq 0 .
$$

Similarly to [2, 3] we derive the equation

$$
x_{\alpha}(t)=y_{\alpha}(t)+\Delta_{\alpha}\left[x_{\alpha}\right](t)+x_{\alpha} * G_{\alpha}(t)
$$

from (10) by substituting $F_{\alpha}(t, d s)=\Delta_{\alpha}(t, d s)+G_{\alpha}(d s)$.

Now we introduce the following functions, kernel, and measure:

$$
\begin{gathered}
\bar{x}_{\alpha}(t)=\exp (\beta t) x_{\alpha}(t), \quad \bar{y}_{\alpha}(t)=\exp (\beta t) y_{\alpha}(t), \\
\bar{\Delta}_{\alpha}(t, d s)=\exp (\beta s) \Delta_{\alpha}(t, d s), \quad \bar{G}_{\alpha}(d s)=\exp (\beta s) G_{\alpha}(d s) .
\end{gathered}
$$

According to Remark 1 of [3], we derive the equation

$$
\bar{x}_{\alpha}(t)=\bar{y}_{\alpha}(t)+\bar{\Delta}_{\alpha}\left[\bar{x}_{\alpha}\right](t)+\bar{x}_{\alpha} * \bar{G}_{\alpha}(t)
$$

from (44) by multiplying it by the function $\exp (\beta t)$.

In view of definitions (13) and (14), $\bar{G}_{\alpha}$ is a probability measure. Thus one can apply Theorem 1 for equation (46) with the distributions $F_{\alpha}=\bar{G}_{\alpha}$.

The inclusion $\left(\bar{G}_{\alpha}\right) \subset \mathrm{L}(\nu, m) \cap \mathrm{M}(l, 1)$ follows by condition (17).

The mean values are such that

$$
m_{\alpha}=\int_{0}^{\infty} s \bar{G}_{\alpha}(d s)=\int_{0}^{\infty} e^{\beta s} s G_{\alpha}(d s)=\hat{g}_{\alpha}^{\prime}(\beta)=\mu_{\alpha} .
$$

In particular,

$$
\inf _{\alpha} m_{\alpha}=\inf _{\alpha} \mu_{\alpha}>0
$$

by condition (17) and according to (41).

Finally, the assumption of Theorem 1 imposed on $\left(y_{\alpha}\right)$ is reduced to the inclusion

$$
\left(\left|\bar{y}_{\alpha}\right|\right)+\left(\left|\bar{\Delta}_{\alpha}\left[\bar{x}_{\alpha}\right]\right|\right) \subset \mathrm{B}_{0}^{1}\left(\left\{v_{\alpha}\right\},\left\{t_{\alpha}\right\}\right) \cap \mathrm{L}_{0}^{1}\left(\left\{v_{\alpha}\right\},\left\{t_{\alpha}\right\}\right) .
$$

Note that the values $y_{\alpha}(t)$ on the right hand side of (48) are considered only for $t \leq t_{\alpha}$ by definitions (3) and (4). Since $\exp (\beta t) \leq \exp \left(\beta t_{\alpha}\right) \rightarrow \exp (u)<\infty$ and

$$
\begin{gathered}
\left|\bar{y}_{\alpha}(t)\right| \leq \exp \left(\beta t_{\alpha}\right)\left|y_{\alpha}(t)\right|, \\
\left|\bar{\Delta}_{\alpha}\left[\bar{x}_{\alpha}\right](t)\right| \leq \exp \left(\beta t_{\alpha}\right) \delta_{\alpha}(t) \sup _{\alpha>0, t \geq 0}\left|x_{\alpha}(t)\right|,
\end{gathered}
$$

inclusion (48) follows from (18), (19), (3), and (44). 
Thus Theorem 1 yields the convergence

$$
\sup _{v_{\alpha} \leq t \leq t_{\alpha}}\left|\omega_{\alpha}(t)\right|=O\left(\gamma_{\alpha}\right)+o\left(\Gamma_{\alpha}\right) \rightarrow 0, \quad \alpha \rightarrow 0,
$$

in view of (48), (49), and (23), where $\omega_{\alpha}$ equals the argument of the absolute value in (6) for equation (46):

$$
\begin{gathered}
\omega_{\alpha}(t) \equiv \bar{x}_{\alpha}(t)-u_{\alpha}(t)-v_{\alpha}(t), \\
u_{\alpha}(t) \equiv \mu_{\alpha}^{-1} \int_{0}^{t} \bar{y}_{\alpha}(s) d s, \\
v_{\alpha}(t) \equiv \mu_{\alpha}^{-1} \int_{0}^{t} \bar{\Delta}_{\alpha}\left[\bar{x}_{\alpha}\right](s) d s .
\end{gathered}
$$

Using (51) we express $\bar{x}_{\alpha}$ via $\omega_{\alpha}$ and substitute the result into (46) under the sign of the operator $\bar{\Delta}_{\alpha}$ :

$$
\bar{x}_{\alpha}(t)=\bar{y}_{\alpha}(t)+z_{\alpha}(t)+q_{\alpha}(t)+r_{\alpha}(t)+\bar{x}_{\alpha} * \bar{G}_{\alpha}(t),
$$

where

$$
z_{\alpha}(t) \equiv \bar{\Delta}_{\alpha}\left[u_{\alpha}\right](t), \quad q_{\alpha}(t) \equiv \bar{\Delta}_{\alpha}\left[v_{\alpha}\right](t), \quad r_{\alpha}(t) \equiv \bar{\Delta}_{\alpha}\left[\omega_{\alpha}\right](t) .
$$

Now we apply Theorem 1 to equation (52).

The inclusions $\left(\bar{G}_{\alpha}\right) \subset \mathrm{L}(\nu, m) \cap \mathrm{M}(l, 1)$ hold by condition (17), while the inclusion

$$
\left(\bar{y}_{\alpha}\right) \subset \mathrm{B}_{0}^{1}\left(\left\{v_{\alpha}\right\},\left\{t_{\alpha}\right\}\right) \cap \mathrm{L}_{0}^{1}\left(\left\{v_{\alpha}\right\},\left\{t_{\alpha}\right\}\right)
$$

is proved in (48).

Now we obtain from (52) and (45) that

$$
\begin{aligned}
z_{\alpha}(t) & =\mu_{\alpha}^{-1} \int_{0}^{t} \exp (\beta s) \Delta_{\alpha}(t, d s) \int_{0}^{t-s} \exp (\beta u) y_{\alpha}(u) d u \\
& =\mu_{\alpha}^{-1} \int_{0}^{t} \exp (\beta u) y_{\alpha}(u)\left[\int_{0}^{t-u} \exp (\beta s) \Delta_{\alpha}(t, d s)\right] d u
\end{aligned}
$$

Thus

$$
\begin{aligned}
\left|z_{\alpha}(t)\right| & \leq \exp \left(\beta t_{\alpha}\right) \int_{0}^{t}\left|y_{\alpha}(u)\right|\left|\Delta_{\alpha}\right|(t,[0, t-u)) d u \\
& \leq \exp \left(\beta t_{\alpha}\right) \delta_{\alpha}(t) \int_{0}^{t_{\alpha}}\left|y_{\alpha}(u)\right| d u
\end{aligned}
$$

for $0 \leq t \leq t_{\alpha}$.

Since $\left(y_{\alpha}\right) \subset \mathrm{L}_{0}^{1}\left(\left\{v_{\alpha}\right\},\left\{t_{\alpha}\right\}\right)$, the integral on the right hand side of (53) is bounded by Definition 5. Hence the inclusion $\left(z_{\alpha}\right) \subset \mathrm{B}_{0}^{1}\left(\left\{v_{\alpha}\right\},\left\{t_{\alpha}\right\}\right) \cap \mathrm{L}_{0}^{1}\left(\left\{v_{\alpha}\right\},\left\{t_{\alpha}\right\}\right)$ follows from inequality (53) and inclusions (18).

Further,

$$
\begin{aligned}
\left|q_{\alpha}(t)\right| & =\mu_{\alpha}^{-1}\left|\int_{0}^{t} e^{\beta s} \Delta_{\alpha}(t, d s) \int_{0}^{t-s} \bar{\Delta}_{\alpha}\left[\bar{x}_{\alpha}\right](u) d u\right| \\
& =\mu_{\alpha}^{-1}\left|\int_{0}^{t} e^{\beta s} \Delta_{\alpha}(t, d s) \int_{0}^{t-s} \int_{0}^{u} \Delta_{\alpha}(u, d v) e^{\beta v} x_{\alpha}(u-v) e^{\beta(u-v)} d u\right| \\
& \leq \mu_{\alpha}^{-1} \exp \left(\beta t_{\alpha}\right) \int_{0}^{t}|\Delta|_{\alpha}(t, d s) \int_{0}^{t-s} \delta_{\alpha}(u) d u \sup _{\alpha, t \leq t_{\alpha}}\left|x_{\alpha}(t)\right| \\
& \leq \mu_{\alpha}^{-1} \exp \left(\beta t_{\alpha}\right) \delta_{\alpha}(t) \sup _{\alpha} \int_{0}^{t_{\alpha}} \delta_{\alpha}(s) d s \sup _{\alpha, t \leq t_{\alpha}}\left|x_{\alpha}(t)\right|
\end{aligned}
$$

for $0 \leq t \leq t_{\alpha}$. 
The integral in the last line of (54) is finite, since $\left(\delta_{\alpha}\right) \subset \mathrm{L}_{0}^{1}\left(\left\{v_{\alpha}\right\},\left\{t_{\alpha}\right\}\right)$. Thus inequality (54) and inclusions (18) imply that $\left(q_{\alpha}\right) \subset \mathrm{B}_{0}^{1}\left(\left\{v_{\alpha}\right\},\left\{t_{\alpha}\right\}\right) \cap \mathrm{L}_{0}^{1}\left(\left\{v_{\alpha}\right\},\left\{t_{\alpha}\right\}\right)$.

Finally,

$$
\left|r_{\alpha}(t)\right|=\mu_{\alpha}^{-1}\left|\int_{0}^{t} e^{\beta s} \Delta_{\alpha}(t, d s) \omega_{\alpha}(t-s)\right| \leq \mu_{\alpha}^{-1} \exp \left(\beta t_{\alpha}\right) \delta_{\alpha}(t) \sup _{\alpha, s \leq t_{\alpha}} \omega_{\alpha}(s),
$$

whence (in a manner as above) we deduce that $\left(r_{\alpha}\right) \subset \mathrm{B}_{0}^{1}\left(\left\{v_{\alpha}\right\},\left\{t_{\alpha}\right\}\right) \cap \mathrm{L}_{0}^{1}\left(\left\{v_{\alpha}\right\},\left\{t_{\alpha}\right\}\right)$. Thus all the assumptions of Theorem 1 hold for equation (52).

Therefore

$$
x_{\alpha}(t)-\mu_{\alpha}^{-1} \int_{0}^{t}\left(\bar{y}_{\alpha}(s)+z_{\alpha}(s)+q_{\alpha}(s)+r_{\alpha}(s)\right) d s=O\left(\gamma_{\alpha}\right)+o\left(\Gamma_{\alpha}\right) \rightarrow 0
$$

as $\alpha \rightarrow 0$ uniformly with respect to $v_{\alpha} \leq t \leq t_{\alpha}$. Condition (21) together with the first inequality in (54) implies that

$$
\begin{aligned}
\left|\int_{0}^{t} q_{\alpha}(t) d t\right| & \leq \int_{0}^{t_{\alpha}}\left|q_{\alpha}(t)\right| d t \leq C \int_{0}^{t_{\alpha}} \int_{0}^{t}\left|\Delta_{\alpha}\right|(t, d s) \int_{0}^{t-s} \delta_{\alpha}(u) d u d t \\
& =C \int_{0}^{t_{\alpha}} \delta_{\alpha}(u) d u \int_{u}^{t_{\alpha}}\left|\Delta_{\alpha}\right|(t,[0, t-u)) d t=O\left(\varepsilon_{\alpha}\right) \rightarrow 0, \quad \alpha \rightarrow 0,
\end{aligned}
$$

for $v_{\alpha} \leq t \leq t_{\alpha}$.

Similarly, (55) and condition (20) imply that

$$
\begin{aligned}
\int_{0}^{t_{\alpha}}\left|r_{\alpha}(t)\right| d t \leq & \mu_{\alpha}^{-1} \int_{0}^{t_{\alpha}}\left|\int_{0}^{t-v_{\alpha}} e^{\beta s} \Delta_{\alpha}(t, d s) \omega_{\alpha}(t-s)\right| d t \\
& +\mu_{\alpha}^{-1} \int_{0}^{t_{\alpha}}\left|\int_{t-v_{\alpha}}^{t} e^{\beta s} \Delta_{\alpha}(t, d s) \omega_{\alpha}(t-s)\right| d t \\
\leq & \mu_{\alpha}^{-1} \exp \left(\beta t_{\alpha}\right) \int_{0}^{t_{\alpha}} \delta_{\alpha}(t) d t \sup _{v_{\alpha} \leq s \leq t_{\alpha}} \omega_{\alpha}(s) \\
& +\mu_{\alpha}^{-1} \exp \left(\beta t_{\alpha}\right) \int_{0}^{t_{\alpha}}\left|\Delta_{\alpha}\right|\left(\left[t-v_{\alpha}, t\right)\right) d t \sup _{\alpha, s \leq t_{\alpha}} \omega_{\alpha}(s) \\
= & O\left(\gamma_{\alpha}\right)+o\left(\Gamma_{\alpha}\right)+O\left(\rho_{\alpha}\right) \rightarrow 0, \quad \alpha \rightarrow 0 .
\end{aligned}
$$

Taking into account (53) and (50) we derive (22) from (56).

To prove Remark 2 one can use relation (4) of Lemma 1 in [3] with the constant function $w(t)$ being equal to the left hand side of (24).

Proof of Corollary 1. The proof is reduced to the substitutions

$$
\Delta_{\alpha}(t, B) \equiv 0 \quad \text { and } \quad \delta_{\alpha}(t) \equiv 0
$$

in Theorem 3.

Proof of Theorem 4. We apply Theorem 3 to equation (25) and take into account (29) and (30).

Cramér's condition (13) follows from (28), (34), and (32):

$$
\int_{0}^{\infty} \exp (\delta x) \bar{G}_{\alpha}(d x)=\left(1-\theta_{\alpha}\right) \widehat{G}(\beta+\delta)>\left(1-\theta_{\alpha}\right) \widehat{G}(\beta)=1 .
$$

The inclusion $\left(\bar{G}_{\alpha}, \alpha>0\right) \subset \mathrm{L}(\nu, m)$ follows from Definition 1, since

$$
\bar{G}_{\alpha}^{* m}(B)=\int_{B} \exp (\beta s) G_{\alpha}^{* m}(d s) \geq G_{\alpha}^{* m}(B) \geq \nu(B) .
$$


Further, the inclusion $\left(\bar{G}_{\alpha}, \alpha>0\right) \subset \mathrm{M}(l, 1)$ is a consequence of Cramér's condition (32), since $\bar{G}_{\alpha}(d s) \leq \exp (\beta s) G(d s)$, where $\beta \rightarrow 0$.

We use Theorem 3 with the functions

$$
v_{\alpha} \equiv 0 .
$$

Then we prove the inclusion

$$
\left(\nu_{\alpha}(t)\right) \subset \mathrm{B}_{0}^{1}\left(\{0\},\left\{t_{\alpha}\right\}\right) \cap \mathrm{L}_{0}^{1}\left(\{0\},\left\{t_{\alpha}\right\}\right) .
$$

Indeed, the first condition in (3) follows from (36), while the second condition there holds, since $\nu_{\alpha}(t)$ is bounded, namely $\left|\nu_{\alpha}(t)\right| \leq 1$.

Further, the first condition in (4) follows from Remark 1 with functions $v_{\alpha}$ as in (57), while the second condition is equivalent to (37).

By definitions (27) and (30),

$$
y_{\alpha}(t)=\left(\pi_{\alpha}(t)-\theta_{\alpha}\right) 1_{t \leq b_{\alpha}}+\theta_{\alpha} 1_{t \leq b_{\alpha}}=\nu_{\alpha}(t) 1_{t \leq b_{\alpha}}+\theta_{\alpha} 1_{t \leq b_{\alpha}} .
$$

Hence the inclusion

$$
\left(y_{\alpha}\right) \subset \mathrm{B}_{0}^{1}\left(\{0\},\left\{t_{\alpha}\right\}\right) \cap \mathrm{L}_{0}^{1}\left(\{0\},\left\{t_{\alpha}\right\}\right)
$$

follows from (57), (58), the equality $\theta_{\alpha} 1_{t \leq b_{\alpha}}=0$ for $t>b_{\alpha}$, and from the convergence $\beta b_{\alpha} \rightarrow v<u<\infty$ as $\alpha \rightarrow 0$. Here $\tau_{\alpha}(t) \equiv \min \left(t_{\alpha}-b_{\alpha}, t\right)$. The inclusion

$$
\tau=\left(\tau_{\alpha}(t)\right) \in \Upsilon_{\infty}\left(\left\{t_{\alpha}\right\}\right)
$$

holds, since

$$
\tau_{\alpha}(t)=t_{\alpha}-b_{\alpha} \rightarrow \infty, \quad \alpha \rightarrow 0,
$$

as $\tau_{\alpha}(t)<t$ by the strict inequality $v<u$.

The inclusion $\left(\delta_{\alpha}\right) \subset \mathrm{B}_{0}^{1}\left(\{0\},\left\{t_{\alpha}\right\}\right) \cap \mathrm{L}_{0}^{1}\left(\{0\},\left\{t_{\alpha}\right\}\right)$ is an obvious corollary of (58) and (29). The corresponding functions in (23) are such that $\gamma_{\alpha}=O\left(\psi_{\alpha}\right)$ and $\Gamma_{\alpha}=O\left(\phi_{\alpha}\right)$ as $\alpha \rightarrow 0$ in view of (29), (37), and (36).

Condition (20) follows from (57), since $\left|\Delta_{\alpha}\right|(t,[t, t))=0$.

Similarly, condition (21) holds by (37) in view of (29):

$$
\begin{aligned}
\varepsilon_{\alpha} & =\int_{0}^{t_{\alpha}}\left|\nu_{\alpha}(s)\right| G(s) d s \int_{s}^{t_{\alpha}}\left|\nu_{\alpha}(t)\right| G(t-s) d t \\
& \leq \int_{0}^{t_{\alpha}}\left|\nu_{\alpha}(t)\right| \int_{0}^{t}\left|\nu_{\alpha}(s)\right| d s d t=\frac{1}{2}\left(\int_{0}^{t_{\alpha}}\left|\nu_{\alpha}(t)\right| d t\right)^{2} \\
& =O\left(\phi_{\alpha}^{2}\right), \quad \alpha \rightarrow 0 .
\end{aligned}
$$

Finally, $x_{\alpha}(t, b)$ is the probability of a random event, that is,

$$
x_{\alpha}(t, b) \in[0,1],
$$

whence $0 \leq \bar{x}_{\alpha}(t) \leq \exp (\beta t) \leq \exp \left(\beta t_{\alpha}\right) \rightarrow \exp (u)<\infty$ and boundedness (19) follows.

Further $\mu_{\alpha} \rightarrow \mu=\mathrm{E} \xi_{1}$ by (47). Now we obtain from (22) that

$$
e^{\beta t_{\alpha}} x_{\alpha}\left(t_{\alpha}, b_{\alpha}\right)-h_{\alpha}=O\left(\psi_{\alpha}+\phi_{\alpha}^{2}\right)+O\left(\phi_{\alpha}\right) \rightarrow 0, \quad \alpha \rightarrow 0,
$$


where, by (27), (29), (30), and (59),

$$
\begin{aligned}
h_{\alpha} \equiv & \mu_{\alpha}^{-1} \int_{0}^{t_{\alpha}}\left[e^{\beta t} y_{\alpha}(t)+\mu_{\alpha}^{-1} \int_{0}^{t} e^{\beta s} y_{\alpha}(s) \int_{0}^{t-s} e^{\beta r} \Delta_{\alpha}(t, d r) d s\right] d t \\
= & \mu_{\alpha}^{-1} \int_{0}^{t_{\alpha}}\left[e^{\beta t} \pi_{\alpha}(t) 1_{t \leq b_{\alpha}}+\mu_{\alpha}^{-1} \int_{0}^{t} e^{\beta s} \pi_{\alpha}(s) 1_{s \leq b_{\alpha}}\right. \\
& \left.\times \int_{0}^{t-s} e^{\beta r}\left(\theta_{\alpha}-\pi_{\alpha}(t)\right) G(d r) d s\right] d t \\
= & \mu_{\alpha}^{-1}\left(e^{\beta b_{\alpha}}-1\right) \beta^{-1} \theta_{\alpha}+\mu_{\alpha}^{-1} \int_{0}^{t_{\alpha}} \nu_{\alpha}(t) e^{\beta t} 1_{t \leq b_{\alpha}} d t \\
& -\mu_{\alpha}^{-2} \int_{0}^{t_{\alpha}}\left[\int_{0}^{t} e^{\beta s} \theta_{\alpha} 1_{s \leq b_{\alpha}} \int_{0}^{t-s} e^{\beta r} \nu_{\alpha}(t) G(d r) d s\right] d t \\
& -\mu^{-2} \theta_{\alpha} \beta^{-1} \int_{0}^{u}\left[\int_{0}^{t} e^{s} 1_{s \leq v} \int_{0}^{(t-s) / \beta} e^{\beta r} \nu_{\alpha}(t / \beta) \beta^{-1} G(d r) d s\right] d t .
\end{aligned}
$$

The first term on the right hand side is equivalent to $\mu^{-1}(\exp (v)-1) \mu+O(\beta)$ by convergence (35). Since $\beta t_{\alpha} \rightarrow u>0, \beta b_{\alpha} \rightarrow v>0$, and since the integrand is bounded, we scale the variables by $\beta^{-1}$ and prove that the right hand side of (61) is asymptotically equivalent to

$$
\begin{aligned}
e^{v}- & +\mu^{-1} \int_{0}^{u} \nu_{\alpha}(t / \beta) \beta^{-1} e^{t} 1_{t \leq v} d t \\
& -\mu^{-2} \theta_{\alpha} \beta^{-1} \int_{0}^{u}\left[\int_{0}^{t} e^{s} 1_{s \leq v} \int_{0}^{(t-s) / \beta} e^{\beta r} \nu_{\alpha}(t / \beta) \beta^{-1} G(d r) d s\right] d t \\
& +O(\beta)+O\left(\phi_{\alpha}^{2}\right)
\end{aligned}
$$

in view of (37). Here we used the following upper bound for the last term on the right hand side of (61):

$$
\mu_{\alpha}^{-2} \exp \left(\beta t_{\alpha}\right) \int_{0}^{t_{\alpha}}\left|\nu_{\alpha}(t)\right| \int_{0}^{t}\left|\nu_{\alpha}(s)\right| d s d t=O\left(\phi_{\alpha}^{2}\right), \quad \alpha \rightarrow 0 .
$$

Applying the dominated convergence theorem we conclude that

$$
\int_{0}^{(t-s) / \beta} e^{\beta r} G(d r)-1=O(\beta), \quad \beta \rightarrow 0,
$$

whence

$$
\begin{aligned}
h_{\alpha}- & e^{v}+1-\mu^{-1} \int_{0}^{v} \nu_{\alpha}(t / \beta) \beta^{-1} e^{t} d t+\mu^{-1} \int_{0}^{u} \nu_{\alpha}(t / \beta) \beta^{-1} \int_{0}^{t} e^{s} 1_{s \leq v} d s d t \\
& =O(\beta)+O\left(\phi_{\alpha}^{2}\right)
\end{aligned}
$$

as $\alpha \rightarrow 0$. This proves (38).

Proof of Example 1. The expressions in (36) and (37) do not exceed

$$
\psi_{\alpha}=\theta_{\alpha} \sup _{s \geq 0}|l(s)|=O\left(\theta_{\alpha}\right) \rightarrow 0, \quad \alpha \rightarrow 0,
$$

and, respectively,

$$
\phi_{\alpha}=\theta_{\alpha} \int_{0}^{t_{\alpha}}|l(s)| d s=O\left(\theta_{\alpha}\right) \rightarrow 0, \quad \alpha \rightarrow 0
$$


Proof of Example 2. The expressions in (36) and (37) do not exceed

$$
\psi_{\alpha}=\varkappa_{\alpha} \beta \sup _{t \geq 0}|l(\beta t)|=o\left(\varkappa_{\alpha}\right), \quad \beta \rightarrow 0
$$

and, respectively,

$$
\phi_{\alpha}=x_{\alpha} \beta \int_{0}^{t_{\alpha}}|l(\beta s)| d s=O\left(\varkappa_{\alpha}\right), \quad \beta \rightarrow 0 .
$$

\section{BIBLIOGRAPHY}

1. N. V. Kartashov, Strong Stable Markov Chains, VSP/TViMS Scientific Publishers, Utrecht/Kiev, 1996. MR1451375 (99e:60150)

2. M. V. Kartashov, Inhomogeneous perturbations of a renewal equation and the Cramér-Lundberg theorem for a risk process with variable premium rates, Teor. Imovir. Mat. Stat. 78 (2008), 55-66; English transl. in Theory Probab. Math. Statist. 78 (2009), 61-73. MR2446849 (2010a:60295)

3. M. V. Kartashov, Boundedness, limits, and stability of solutions of renewal equation with a nonhomogeneous perturbation on the semiaxis, Teor. Imovir. Mat. Stat. 81 (2009), 65-75; English transl. in Theory Probab. Math. Statist. 81 (2010), 71-83. MR2667311 (2011f:60154)

4. D. C. M. Dickson, The probability of ultimate ruin with a variable premium loading - a special case, Scand. Actuarial J. (1991), 75-86.

5. H. Gerber, On the probability of ruin in the presence of a linear dividend barrier, Scand. Actuarial J. (1981), 105-115. MR623405 (83c:62169)

6. H. Gerber and E. S. W. Shiu, On the time value of ruin, Proc. 31 Actuarial Research Conference, Ball Statte Univ., August 1996, pp. 145-199.

7. H. Schmidli, An extension to the renewal theorem and an application to risk theory, Ann. Appl. Probab. 7 (1997), no. 1, 121-133. MR1428752 (97k:60234)

8. G. C. Tailor, Probability of ruin with variable premium rate, Scand. Actuarial J. (1980), 57-76. MR.578447 (81m:62188)

9. N. V. Kartashov, On ruin probabilities for a risk process with bounded reserves, Teor. Imovir. Mat. Stat. 60 (1999), 46-58; English transl. in Theor. Probab. Math. Stat. 60 (2000), 53-65. MR.1826141

10. M. V. Kartashov and O. M. Stroyev, The Lundberg approximation for the risk function in an almost homogeneous environment, Teor. Imovir. Mat. Stat. 73 (2005), 63-71; English transl. in Theor. Probab. Math. Stat. 73 (2006), 71-79. MR2213842 (2007b:62121)

11. M. V. Kartashov, Stability of transient quasihomogeneous Markov semigroups and an estimate for ruin probability, Teor. Imovir. Mat. Stat. 75 (2006), 36-44; English transl. in Theor. Probab. Math. Stat. 75 (2007), 41-50. MR2321179 (2008d:60100)

12. N. V. Kartashov, Equivalence of uniform renewal theorems and their criteria, Teor. Veroyatnost. i Mat. Statist. 27 (1982), 51-60; English transl. in Theor. Probab. Math. Stat. 27 (1983), 55-64. MR673349 (83m:60114)

13. N. V. Kartashov, A generalization of the Stone representation and necessary conditions for uniform convergence in the renewal theorem, Teor. Veroyatnost. i Mat. Statist. 26 (1982), 49-62; English transl. in Theor. Probab. Math. Stat. 26 (1983), 53-67. MR664903(83m:60113)

14. W. Feller, An Introduction to Probability Theory and its Applications, vol. 2, John Wiley \& Sons, New York, 1966. MR0210154 (35:1048)

15. A. A. Borovkov, Stochastic Processes in Queueing Theory, Nauka, Moscow, 1972; English transl., Springer-Verlag, New York-Berlin, 1976. MR 0315800 (47:4349)

16. C. Stone, On absolutely continuous components and renewal theory, Ann. Math. Stat. 37 (1966), 271-275. MR0196795 (33:4981)

17. V. M. Shurenkov, Ergodic Theorems and Related Problems, Nauka, Moscow, 1989; English transl., VSP, Utrecht, 1998. MR1690361(2000i:60002)

Department of Probability Theory, Statistics, and Actuarial Mathematics, Faculty for Mechanics and Mathematics, National Taras Shevchenko University, Academician Glushkov Avenue 2, Kiev 03127, Ukraine

E-mail address: nkartashov@skif.com.ua

Received 11/JAN/2010

Translated by S. KVASKO 